\title{
Genome-wide sequencing-based identification of methylation quantitative trait loci and their role in schizophrenia risk
}

\author{
Kira A. Perzel Mandell (1) 1,2, Nicholas J. Eagles (10) 1, Richard Wilton ${ }^{3}$, Amanda J. Price (1) 1,2, Stephen A. Semick (1) 1, \\ Leonardo Collado-Torres (1) 1, William S. Ulrich1, Ran Tao', Shizhong Han ${ }^{1,4}$, Alexander S. Szalay 3,5,

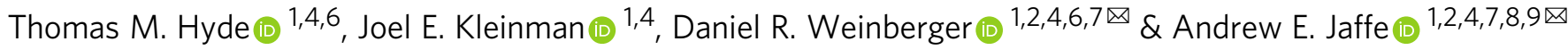

DNA methylation (DNAm) is an epigenetic regulator of gene expression and a hallmark of gene-environment interaction. Using whole-genome bisulfite sequencing, we have surveyed DNAm in 344 samples of human postmortem brain tissue from neurotypical subjects and individuals with schizophrenia. We identify genetic influence on local methylation levels throughout the genome, both at $\mathrm{CpG}$ sites and $\mathrm{CpH}$ sites, with $86 \%$ of SNPs and 55\% of $\mathrm{CpGs}$ being part of methylation quantitative trait loci (meQTLs). These associations can further be clustered into regions that are differentially methylated by a given SNP, highlighting the genes and regions with which these loci are epigenetically associated. These findings can be used to better characterize schizophrenia GWAS-identified variants as epigenetic risk variants. Regions differentially methylated by schizophrenia risk-SNPs explain much of the heritability associated with risk loci, despite covering only a fraction of the genomic space. We provide a comprehensive, single base resolution view of association between genetic variation and genomic methylation, and implicate schizophrenia GWASassociated variants as influencing the epigenetic plasticity of the brain.

\footnotetext{
${ }^{1}$ Lieber Institute for Brain Development, Johns Hopkins Medical Campus, Baltimore, MD, USA. ${ }^{2}$ Department of Genetic Medicine, Johns Hopkins University School of Medicine (JHSOM), Baltimore, MD, USA. ${ }^{3}$ Department of Physics and Astronomy, Johns Hopkins University, Baltimore, MD, USA. ${ }^{4}$ Department of Psychiatry and Behavioral Sciences, JHSOM, Baltimore, MD, USA. ${ }^{5}$ Department of Computer Science, JHSOM, Baltimore, MD, USA. ${ }^{6}$ Department of Neurology, JHSOM, Baltimore, MD, USA. ${ }^{7}$ Department of Neuroscience, JHSOM, Baltimore, MD, USA. 8 Department of Mental Health, Johns Hopkins Bloomberg School of Public Health (JHBSPH), Baltimore, MD, USA. ${ }^{9}$ Department of Biostatistics, JHBSPH, Baltimore, MD, USA.

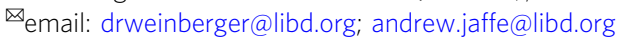


D NA methylation (DNAm) plays an important role in the epigenetic regulation of gene expression. It varies throughout development and among tissue types, and has been thought to be a high-fidelity representation of the interaction between genes and environment. While some variation in DNAm can be attributed to developmental and exogenous factors, such as $\operatorname{diet}^{1}$ and cigarette smoking ${ }^{2}$, Davies et al. ${ }^{3}$ identified some inter-individual variation that is consistent across tissue types. This provided evidence that DNA sequence drives DNA methylation levels, at sites known as methylation quantitative trait loci (meQTLs). Inter-individual DNAm differences have since been confirmed by twin studies ${ }^{4,5}$. Initial studies found methylation association with sequence variants at specific loci6. These epigenetic associations likely extend beyond losing the "C" or "G" allele in CpG dinucleotides (for example, through deamination of the cytosine base in this genomic context $)^{7}$.

Genome-wide studies are necessary to fully understand the extent and genomic properties of meQTLs. However, so far, most large-scale studies have used microarray technologies that only measure a small proportion of $\mathrm{CpGs}^{8-10}$. The largest study to date to test associations between genotype and DNAm used MBD-seq, a method lacking single base-pair resolution ${ }^{11}$. Yet even with limited resolution, these initial studies have found that local genetic influence on DNAm is extensive throughout the genome, and meQTLs are enriched at regulatory sites ${ }^{12,13}$.

Currently, a major puzzle in the field of functional genomics is understanding the molecular effects of genetic risk loci and variants identified by genome-wide association studies (GWAS) for many common disorders and traits which do not involve coding sequences. This is particularly challenging in tissues like brain that are difficult to access or model, leaving little clarity into genetic mechanisms behind psychiatric disorders such as schizophrenia (SCZD). While schizophrenia is highly heritable ${ }^{14}$, and GWAS have identified a growing number of significant loci ${ }^{15,16}$, only few loci have been functionally resolved ${ }^{17}$. Genome-wide gene expression QTL (eQTL) approaches ${ }^{18,19}$, and their extensions ${ }^{20-22}$, have prioritized variants and associated genes, but many genomic loci fail to associate with nearby gene expression. In contrast, associating schizophrenia risk variants with a stable epigenetic mark like DNAm provide clues for potential epigenetic mechanisms of action ${ }^{23,24}$. Indeed, previous meQTL maps using microarray technologies implicated a larger number of SCZD risk loci than eQTL maps, while only measuring a fraction of the methylome ${ }^{10}$. DNAm itself may further reflect the cumulative effects of environmental exposures across the lifespan ${ }^{25}$, and may represent a surrogate of " $\mathrm{E}$ " in GxE interactions that contribute to risks for many disorders ${ }^{26}$ that can further act as a mediator of genetic risk on gene expression.

Unlike microarray technologies, whole-genome bisulfite sequencing (WGBS) has the advantage of measuring cytosine methylation at single base-pair resolution, as well as measuring $\mathrm{CpH}(\mathrm{H}=\mathrm{A}, \mathrm{T}$, or $\mathrm{C})$ DNA methylation levels (in addition to $\mathrm{CpGs}$ ). While $\mathrm{CpH}$ sites are generally unmethylated in somatic tissues, neurons in the human brain have uniquely high levels of $\mathrm{CpHm}^{27}$. By leveraging this technology, we have created the most extensive genomic meQTL map in human postmortem brain tissue to date, and use this information to fine-tune our understanding of the molecular mechanisms of genetic and epigenetic risk for schizophrenia.

\section{Results}

Components of global variation in large-scale WGBS data sets. We performed whole-genome bisulfite sequencing (WGBS) to gain a comprehensive view of genetic influence on DNAm in the adult human brain using two brain regions: the hippocampus and the dorsolateral prefrontal cortex (DLPFC). These regions have been prominently implicated in the pathogenesis of many psychiatric disorders, particularly schizophrenia ${ }^{28}$. After data processing and quality control (see "Methods" section), we analyzed 165 DLPFC samples and 179 hippocampal samples from a total of 183 adult donors aged 18-96 years (161 donors had data from both regions, Supplementary Dataset 1). Data were generated across two large diagnosis- and region-balanced batches. We assessed 29,401,795 CpG sites across the epigenome, with an average post-processing coverage of 17.3 reads per $\mathrm{CpG}$ site. $78 \%$ of sites were highly $(>80 \%)$ methylated while a minority were lowly or unmethylated ( $8 \%$ are $<20 \%$, Supplementary Fig. S1). While the technical effects of measuring DNAm levels using microarrays are well-established ${ }^{29}$, particularly in human brain tissue $^{10}$, corresponding assessments using WGBS data have been limited due to available comparisons being relatively small studies. We, therefore, assessed the contributions of different biological and technical variables on genome-wide CpG DNAm levels measured with WGBS.

First, we performed principal component analysis (PCA) across the raw DNAm levels of the million most variable CpGs. The top principal components were associated with quantitative/genotypedefined ancestry (PC1: 6.5\% variance explained, Supplementary Fig. S2), estimated neuronal fraction (PC2: 3.34\%), processing batch (PC4: $1.37 \%$ ), and brain region (PC5: $0.84 \%$ ). The major batch effects resulted from the inclusion of ENCODE "blacklist" regions ${ }^{30}$, which have been reported to cause problems with mapping and alignment in high-throughput sequencing data, particularly epigenomic data. These processing issues are likely further exacerbated in WGBS data, where the bisulfite treatment results in lower complexity libraries depleted of cytosines, which presumably relates to the influence of blacklist regions and ancestry on DNAm levels. Cytosines in these black-listed regions were therefore removed from reported site-specific analysis results. Another increasingly common step in WGBS data processing involves "smoothing" local CpG DNAm levels within each sample to improve precision and borrow strength across nearby $\mathrm{CpGs}^{31}$. Smoothing reordered the top components of variation (Supplementary Fig. S3), and resulted in the top component of variation representing both batch and estimated neuronal fraction (both PC1 and PC2, explaining $13.9 \%$ and $10 \%$ of the variance, respectively). Previous analyses of Illumina microarray-derived adult homogenate DLPFC data suggested that estimated neuronal fraction was the largest component of (CpG) DNAm level variation ${ }^{10}$. Microarray technology implicitly produces somewhat smooth DNAm levels for a large fraction of probes that target multiple $\mathrm{CpG}$ sites. While the effects of the brain region were further magnified with smoothing, the effects of quantitative ancestry were dampened, and became associated with PC4 (1\% variance explained) rather than PC1 of raw DNAm levels (6\% explained variance; Fig. 1a-e).

We further complemented these global analyses using sitespecific variance components analysis, estimating the percentage of smoothed DNAm level variance explained by technical and biological components at each autosomal $\mathrm{CpG}$, excluding the blacklist ( $N=26,416,185$, using ANOVA, see "Methods" section, Fig. 1f, see "Data availability" section). The factors that explained the largest components of site-specific variation were technical batch (median: 9.6\% variance explained, interquartile range $3.4-19 \%)$, and related flow cell $(7.9 \%, 6.3-9.6 \%)$ and instrument $(2.9 \%, 2.1-3.2 \%)$ variables, as well as the more biological brain region $(1.5 \%, 0.3-5.9 \%)$, estimated neuronal fraction ( $1 \%$, $0.2-3.8 \%)$ variables. Traditionally considered confounders in postmortem human brain studies-including tissue $\mathrm{pH}$ and postmortem interval (PMI) - had very little influence on sitespecific DNAm levels using WGBS (in line with previous microarray-based analyses $\left.{ }^{10}\right)$. For example, $\mathrm{pH}$ and $\mathrm{PMI}$ 

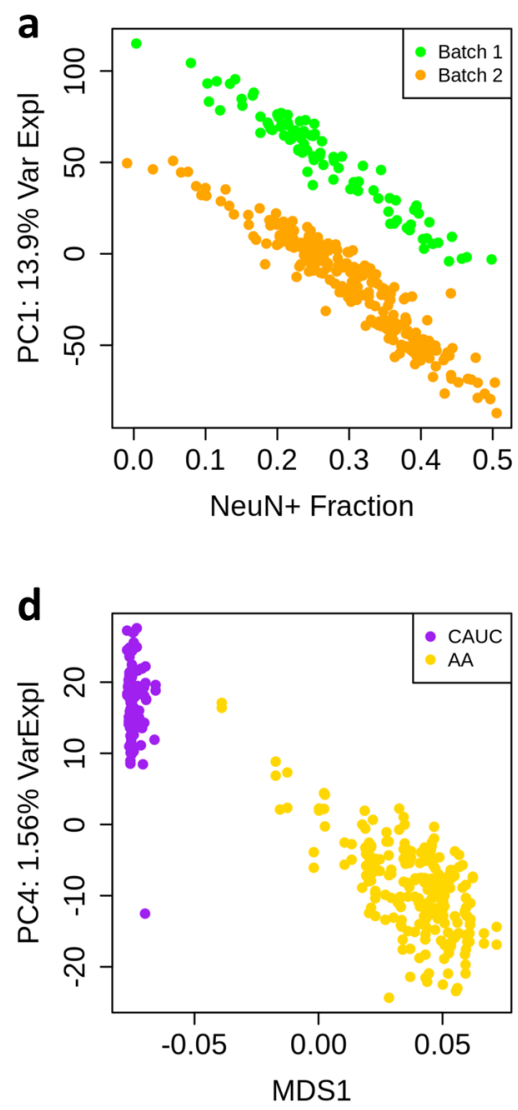

b

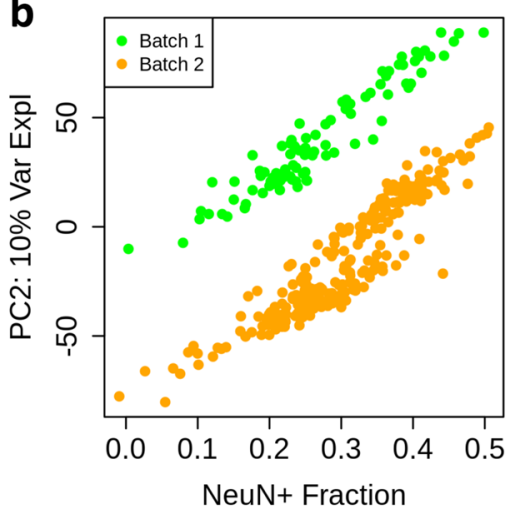

e

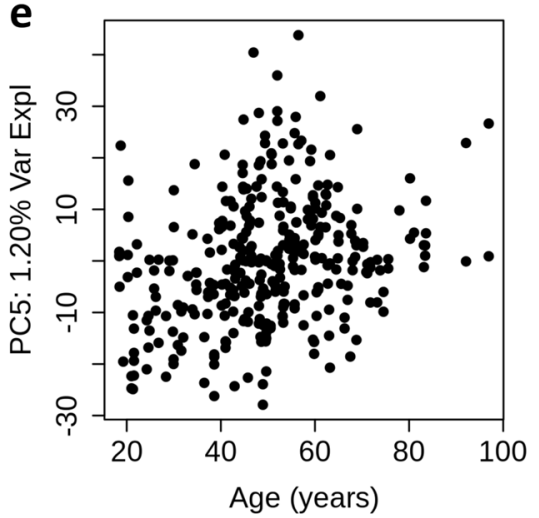

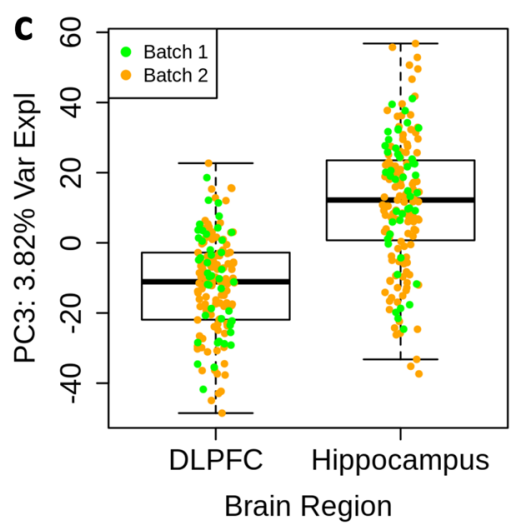

$\mathbf{f}$

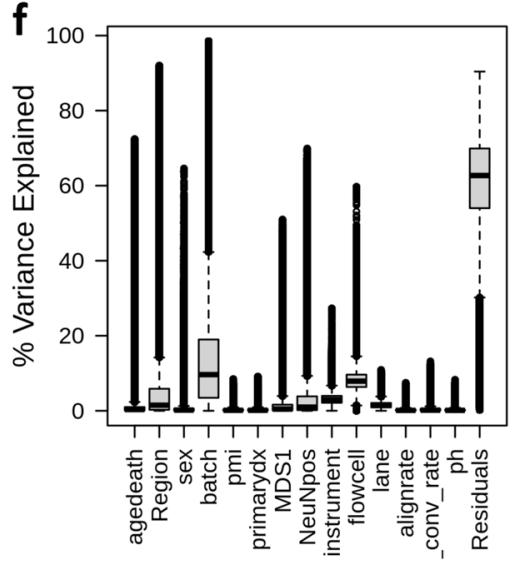

Fig. 1 Variance in smoothed methylation data, post-QC. PCA was performed on all sites excluding the sex chromosomes and ENCODE's blacklist. a, b We find that the top principal components of smoothed methylation data associate with both batch and neuronal composition. $\mathbf{c}$ We see that the third principal component is associated with the brain region, and no longer associated with the batch. d In smoothed methylation data, ethnicity is reduced to the 4th principal component. MDS: multidimensional scaling of genotype data. e Age associates with the fifth principal component. $\mathbf{f} \vee$ ariance explained was analyzed using ANOVA by each individual CpG site. We see that brain region and batch effects explain a large deal of variance, while biological factors such as PMI and pH explain very little. $n=26,416,185$ CpGs.

explained more than $1 \%$ of the variance across only approximately $5 \%$ of measured sites. Other technical variables hypothesized to influence DNAm levels like the sequencing alignment rates and bisulfite conversion rates (estimated with $\lambda$ spike-in sequences, see "Methods" section) showed little influence in this analysis. Overall, there was an extensive residual variation of DNAm levels for the majority of sites beyond these technical and biological variables.

Local genetic variation has strong effects on CpG DNA methylation levels. We hypothesized that a large component of this residual DNAm variation was likely captured by local genetic sequence. We, therefore, performed genome-wide methylation quantitative trait locus (meQTL) analyses (see "Methods" section) on smoothed DNA methylation levels in each brain region separately (across 29,401,795 CpG sites). In the DLPFC, we computed meQTLs between each of these CpGs and the subset of common SNPs within $20 \mathrm{~kb}$ upstream and downstream, which identified 482,579,961 significant SNP-CpG pairs (at FDR $<0.01$, see "Data availability" section), representing 6,807,821 (86\%) of the tested SNPs and 14,551,080 (55\%) of tested CpGs. Sensitivity analyses using the more stringent Bonferroni cutoff (corresponding to $\sim 3$ billion tests) - assuming both CpGs and SNPs are independent (which is likely an overly-stringent assumption given known spatial autocorrelation of $\mathrm{CpGs}$ and linkage disequilibrium of SNPs) - identified 101,482,392 SNP-CpG pairs, representing $15 \%$ of CpGs and $37 \%$ of tested SNPs. Given the high genomic correlation among both CpGs and SNPs, we performed the same analysis with a set of 535,859 LD-independent SNPs $\left(R^{2}<0.2\right)$ to reduce the potential effects of linkage disequilibrium (LD) potentially inflating these statistics. This sensitivity analysis found a substantial proportion of CpGs $(8,390,092,29 \%)$ and SNPs $(402,407,75 \%)$ identified as meQTLs at FDR $<0.01$ with similar properties. Most SNPs associated with methylation levels at many nearby $\mathrm{CpG}$ sites (mean $=57 \mathrm{CpGs}$, median $=43$ CpGs), and the methylation-associated SNPs had varying genomic widths of effect in this local window ranging from $1 \mathrm{bp}$ to the full $40 \mathrm{~kb}$ (mean $=14.5 \mathrm{~kb}$, median $=12.7 \mathrm{~kb})$. Effect sizes were generally small, with a mean of $2.6 \%$ change in methylation level per allele (IQR: $1.4-3.1 \%$ ), but ranging up to $47 \%$. Enforcing more stringent Bonferroni significance among meQTLs resulted in higher effect sizes (mean $=4.5 \%$ ), lower widths of effect (mean $=7.5 \mathrm{~kb}$ ), and fewer-though still numerous-CpGs associated with each SNP (mean = 34). In both analyses we found that SNPs that disrupt a CpG dinucleotide (i.e., a variant at the $\mathrm{C}$ or $\mathrm{G}$, which would ablate the capacity for methylation) have a slightly but significantly lower width of effect and a slightly higher number of correlated CpGs, meaning they have a higher effect density. This may be attributed to the fact that CpGs tend to cluster in the genome. Additionally, despite tested SNPs being LD-independent in the second analysis, we find that half of CpGs associate with more than one SNP, with 
a

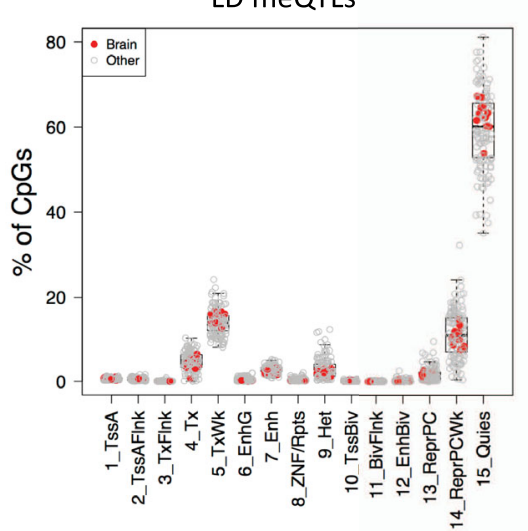

b

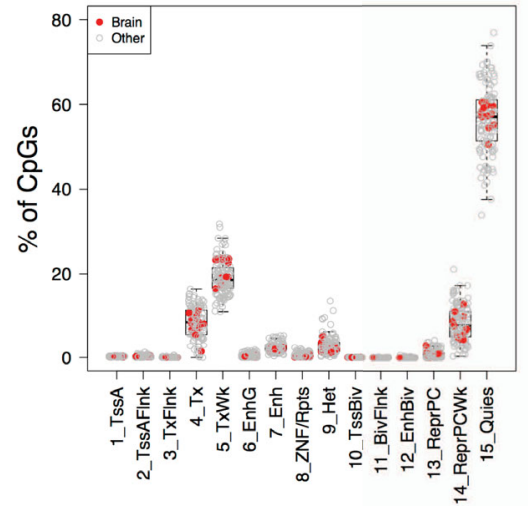

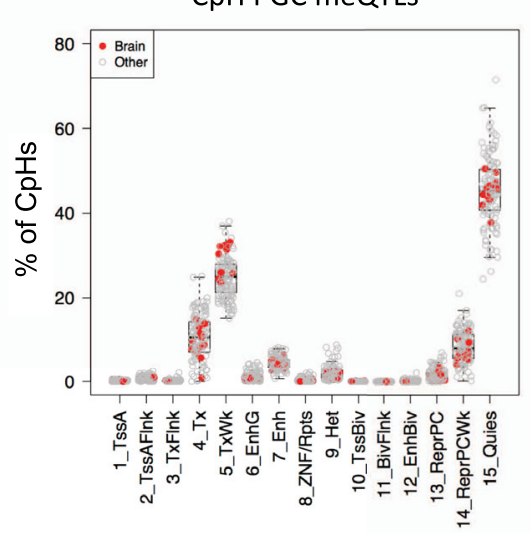

Fig. 2 Chromatin state of meQTLs and gDMRs. We assessed the chromatin state of sites identified as meQTLs and gDMRs using data from various tissues from the Roadmap Epigenomics Project. Data for brain tissues are highlighted in red. $n=127$ tissue types. Chromatin states are defined as follows. 1: Active TSS, 2: Flanking Active TSS, 3: Transcription at gene 5' and 3', 4: Strong transcription, 5: Weak transcription, 6: Genic enhancers, 7: Enhancers, 8: ZNF genes \& repeats, 9: Heterochromatin, 10: Bivalent/Poised TSS, 11: Flanking Bivalent TSS/Enhancer, 12: Bivalent Enhancer, 13: Repressed PolyComb, 14: Weak Repressed PolyComb, 15: Quiescent/Low. a In genome-wide meQTLs, assessing a set of LD-independent SNPs, we see that the vast majority of meQTL-CpGs are in quiescent chromatin regions. b We see that compared to genomic SNPs, CpGs associated with PGC schizophrenia risk SNPs are enriched for regions of active transcription and depleted for regions of quiescent chromatin. $\mathbf{c}$ We see that $\mathrm{CpH}$ sites associated with PGC schizophrenia risk SNPs are often in regions of active transcription.

a mean and median of 2 , in line with previous observations in gene expression data ${ }^{18,32}$. Using information from the Roadmap Epigenome $^{33}$, most genetically-associated CpGs were in quiescent genomic regions, and depleted for enhancer regions in the human brain (Fig. 2a). The meQTLs from both brain regions are searchable by SNPs or genomic regions of interest at: https:// eqtl.brainseq.org/WGBS_meQTL/.

Analogous results were observed in hippocampus samples, yielding 505,142,175 significant pairs that represented 14,647,533 CpGs (55\%) and 6,900,009 SNPs (87\%, see "Data availability" section). Analyses on LD-independent SNPs yielded a nearsimilar proportion of SNPs $(403,373$ SNPs, 77\%) implicating a similar number of CpGs as seen in the DLPFC $(8,566,898)$. Effect sizes were similarly small, with a mean of $2.6 \%$ change in methylation level per allele. Hippocampal meQTLs have similar width of effect as those in DLPFC with a mean of 15,661 bp and an average of $60 \mathrm{CpGs}$ associated with a SNP. These analyses suggested that the global properties of meQTLs were highly similar across brain regions. Given the difficulty in identifying suitable/comparable brain region-specific replication data sets, we treated one region as the discovery data set and the other as replication, and calculated $\pi_{1}$ statistics. By comparing our findings between the two brain regions, we found very high replicability, with hippocampal meQTLs showing a very high sharing $\left(\pi_{1}=0.978\right)$ with significant meQTLs identified in DLPFC (using chr1, see "Methods" section).

We next performed a series of secondary analyses to better characterize the determinants of such extensive genetic regulation of DNA methylation. First, due to the mixed ethnicities of our samples, and the potentially large differences in allele frequencies between ancestry groups ${ }^{34}$, we ran post-hoc meQTL analysis on a subset of meQTLs identified in full genome analysis in the DLPFC, separating samples into two groups by self-reported race. African Americans (AAs) made up 67\% of our total sample, and thus were more likely to drive the results. Using significant meQTLs on chromosome 1, analyses using only African American samples $(N=112)$ showed that $99.91 \%$ of the meQTLs were directionally consistent with the full analysis, with $92 \%$ marginally significant $(P<0.05)$ and $66 \%$ genome-wide significant $(\mathrm{FDR}<0.01)$ in the smaller sample size and an overall sharing of $\pi_{1}=0.995$. In the European ancestry samples $(N=53)$, of the $90 \%$ of meQTLs that had polymorphic SNPs in this group, 95\% of meQTLs were directionally consistent, with $55 \%$ marginally significant and $16 \%$ genome-wide significant with an overall sharing of $\pi_{1}=0.801$. These decreased proportions compared to AA-specific analyses at least partially related to the smaller sample size and resulting in decreased statistical power (Supplementary Fig. S4). We also found that in general, differences in minor allele frequencies across ancestry groups did not associate with differences in meQTL effect magnitude (Supplementary Fig. S5), indicating that differences in ethnicity composition of our samples were likely not driving our combined ancestry analyses above. As an additional sensitivity analysis, we performed meQTL analysis within each diagnostic group separately to assess the extent to which schizophrenia diagnosis influenced our findings. We found very high sharing $\left(\pi_{1}>99 \%\right)$ among significant meQTLs discovered in either diagnostic groups (and assessed in the other/non-discovery group), supporting that meQTL effects were globally similar between diagnostic groups. We further explored the robustness of the selected meQTL window size $(20 \mathrm{~kb})$ using heritability analysis (see "Methods" section) on the methylome ${ }^{35}$ with different window sizes $(20,100$, $500 \mathrm{~kb})$. DNA methylation levels were highly heritable using a $20 \mathrm{~kb}$ window size, with $38 \%$ of tested $\mathrm{CpG}$ sites showing significant heritability $($ FDR $<0.01)$. These heritability results were further consistent with the above meQTL analyses, with $99 \%$ of significantly heritable CpGs being meQTLs (and conversely, $68 \%$ of meQTL-CpGs were heritable). Larger window sizes in heritability analysis actually identified fewer CpGs with significantly heritable methylation, implying that most genetic control of methylation acts in cis and confirming that our meQTL testing window was comprehensive. These WGBS data further replicated meQTLs identified in Illumina 450k microarray data $\left(\pi_{1}=0.67\right)^{10}$, even though CpGs profiled on this microarray platform were depleted for meQTLs ( $30 \%$ of CpGs) compared to WGBS ( $55 \%$ of CpGs), in line with gene-biased designs of Illumina microarrays. We, therefore, identified widespread genetic control of CpG methylation levels. Understanding the details of this landscape may help elucidate the functional significance of SNPs highlighted by GWAS. 
Widespread meQTLs among schizophrenia risk variants. DNA methylation previously has been shown to play a role in mediating genetic risk for neuropsychiatric (and other common) disorders ${ }^{10,36-38}$, but all previous meQTL analyses have utilized microarray, not sequencing-based, methylome data. We performed extensive meQTL analyses on SNPs associated with genetic risk for schizophrenia in these large WGBS data sets. We specifically performed chromosome-scale meQTL analysis using each of the "index" SNPs for loci associated with schizophrenia from the most recent GWAS study of schizophrenia, i.e., PGC2 + CLOZUK ${ }^{15}$. We assessed index SNPs with high-quality genotype data in each region - 152 SNPs in DLPFC and 153 in the hippocampus. Each SNP was tested against every $\mathrm{CpG}$ site in the genome, considering a distance of $<250 \mathrm{~kb}$ cis and everything else trans. In DLPFC we found 25,382 significant (FDR $<0.01$, Supplementary Dataset 2) SNP-CpG pairs, representing 147 SNPs and 25,303 CpGs, showing that most Psychiatric Genomics Consortium (PGC) loci contain SNPs that associate with local DNA methylation levels (as only 107 SNP-CpG pairs were in trans). Schizophrenia risk-associated SNPs on average associated with $172 \mathrm{CpGs}$ (median $=104)$, and in this cis window had an average genomic width of the effect of $177 \mathrm{~kb}$ (median $=147 \mathrm{~kb}$ ).

We further performed functional validation of these associations using corresponding gene expression data. Using RNA-seq data from the same regions and donors (see "Methods" section), we assessed whether methylation at these CpGs correlated with neighboring expression levels. Using previous eQTL analyses on these same PGC loci ${ }^{18,39}$, we assessed the mediation of expression by mCpG (see "Methods" section). Eleven of 127 loci had a correlation between gene expression and the methylation with which they are associated. Importantly, 10 of these associated with at least one $\mathrm{CpG}$ that mediated expression by at least $25 \%$. The same analyses on the exon and junction levels picked up subtler effects, detecting 18 and 27 loci mediating expression levels via methylation, respectively. We found that overall, methylation mediation was most potent on the exon level $($ median $=40 \%)$, then the junction level $($ median $=32 \%)$, and least potent on the full gene level (median $=23 \%$ ), in line with the putative role of DNAm in promoting gene splicing ${ }^{40}$.

The same meQTL analysis was performed in the hippocampus WGBS data, revealing 48,023 significant SNP-CpG pairs (Supplementary Dataset 3), representing 139/153 tested SNPs (including 15,119 trans-meQTLs, 31.5\%). Within the subset of significant DLPFC meQTLs, hippocampal meQTLs had an overall sharing of $\pi_{1}=0.97$, indicating that our findings are very consistent between brain regions.

These results indicated that meQTL effects, at least in the context of GWAS associations with schizophrenia, have much broader effects than traditionally considered, and much wider than the $20 \mathrm{~kb}$ window examined at the full genome level. In order to see if schizophrenia-associated meQTLs are comparable to non-disease-associated meQTLs, we took 5000 random SNPs representing all levels of MAF and ran meQTL analysis with a $250 \mathrm{~kb}$ window. Again we found that the majority of SNPs (93\%) are meQTLs. We also find that neither MAF nor populationMAF differences associate with any meQTL characteristics. Interestingly, we found that these random meQTLs had significantly lower width of effect than schizophrenia-associated meQTLs in both regions (DLPFC $P=8.9 \mathrm{e}-5$, Hippocampus $P=1.1 \mathrm{e}-11$ ), and a significantly fewer number of affected CpGs in the hippocampus $(P=0.002)$. This combined with the chromatin state enrichment analysis below may indicate that these PGC-meQTLs are particularly functional, and potentially involved in disease processes, as opposed to just being standardly representative of the whole genome.
Risk-associated meQTL effects cluster in the genome. We then proceeded to cluster our meQTL-CpGs into genetic differentially methylated regions (gDMRs)-regions where methylation is differential by additive genotype-for better functional characterization. Using a CpG-specific $t$-statistic cutoff of 5 (see "Methods" section), these sites could be clustered into 1277 gDMRs (Fig. 3 and Supplementary Dataset 4 ). The majority of SCZD index SNPs had such gDMRs, and most had more than one (mean $=9.5$, median =6). The overall span of effect for each SNP was much larger than the $20 \mathrm{~kb}$ cis window we tested above for meQTL analyses across the full genome, ranging up to $240 \mathrm{Mb}$ on a single chromosome, with a median of $95 \mathrm{~kb}$ (mean $=17.5 \mathrm{Mb})$. Using Roadmap Epigenome ${ }^{33}$ data, these SCZD risk-associated gDMRs were enriched over the background of genome-wide LD-independent meQTLs for transcriptional and weak transcriptional chromatin signatures (Fig. 2b). They were also comparatively depleted for weak repressive polycomb and quiescent chromatin signatures. Overall, these gDMRs were in or near genes enriched for GO terms related to synapse and membrane potential (Supplementary Fig. S6). 20 gDMRs overlapped with psychENCODE enhancers, and 142 overlapped with promoter regions. The genes connected to these promoters were enriched for GO terms related to acetylcholine, ion channels, and neurotransmitters.

Overall, the results of clustering meQTL-CpGs were quite similar between regions. In the hippocampus, there were 1408 gDMRs (Supplementary Dataset 5), and more than half (853) of gDMRs directly overlapped with a gDMR in the DLPFC. Furthermore, $95.1 \%$ of DLPFC-identified gDMRs showed marginal $P<0.05$ significance among hippocampal meQTL effects (based on the average $P$-value for each gDMR). In general, allelic association with methylation in the hippocampus correlated with the association in DLPFC gDMRs $\left(r^{2}=0.69\right.$, Supplementary Fig. S7). A strong majority of gDMRs were located inside introns. Again we found that most SNPs have multiple associated gDMRs $($ mean $=10$, median $=7)$ and have a high total genomic width $($ mean $=15,447,206 \mathrm{bp}$, median $=122,887 \mathrm{bp}$ ). Only 16 of these gDMRs contain the actual GWAS index SNP, suggesting that these effects are more than just local consequences of genetic variations. Overall, the genes represented by these gDMRs have enrichment for GO terms related to synapses, membrane potential, and inositol triphosphate (IP3), a second messenger signaling molecule. In both regions, a handful of gDMRs had a correlation to the expression of some gene (see "Methods" section), and a few correlated to many genes, but most of these gene-gDMR pairs were on different chromosomes, making results difficult to interpret. Most pairs were negatively correlated though, which fits with the traditional understanding of the suppressive effect of methylation on gene expression ${ }^{41,42}$.

These SCZD risk-associated gDMRs were further used as input to partitioned LDSC analysis (see "Methods" section) to illuminate their clinical relevance ${ }^{43}$. As a first pass, we ran partitioned LDSC analysis on the LD blocks of the schizophrenia GWAS loci used in meQTL analysis, which tested for enrichment of top loci versus the rest of the genome, controlling for CNSrelevant functional loci (see "Methods" section). These significant GWAS loci LD blocks explained $15 \%$ of the additive genetic heritability explained by SNPs $\left(h^{2}{ }_{\mathrm{SNP}}\right)$, with a 10 -fold enrichment over the full genome $(P=8 \mathrm{e}-16)$, in line with being the topranked loci in the GWAS. We then considered two sets of gDMRs defined by two different statistical thresholds: a more liberal $t$ statistic $>3.5$ cutoff (which corresponded roughly to controlling an FDR $<0.01$ in cis meQTL analysis), termed DMRs ${ }^{3.5}$, and the subset of entirely-contained gDMRs defined by $t>5$ (from above), termed DMRs ${ }^{5}$. The DMRs ${ }^{3.5}$ were generally larger and more distant from each index SNP than the DMRs ${ }^{5}$, with the 

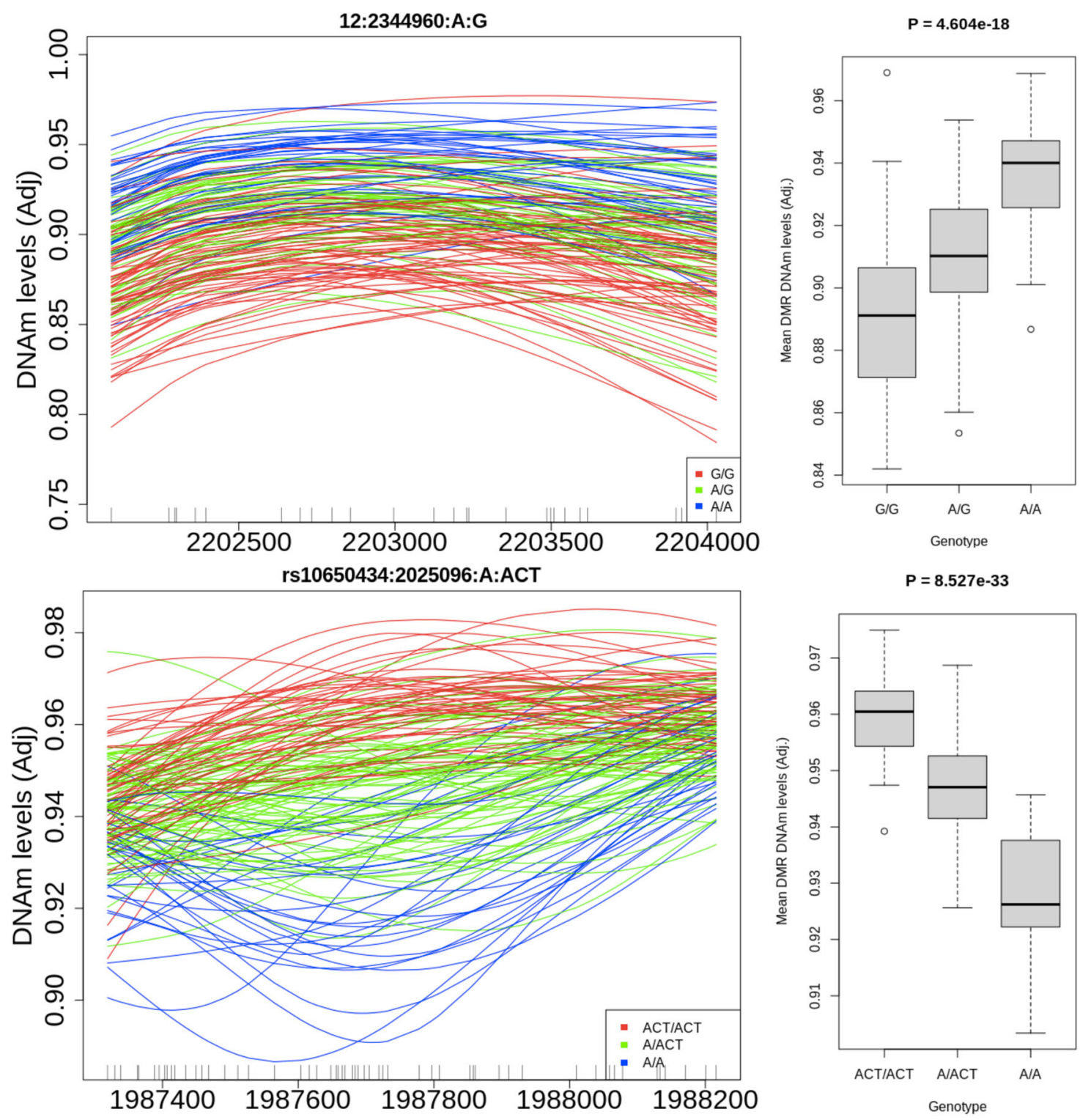

Fig. 3 Schizophrenia risk-associated gDMRs. Two examples of regions where methylation levels are associated with genotype at a schizophrenia risk associated SNP.

majority of DMRs ${ }^{3.5}$ distal (in trans). We further divided these gDMR sets into those cis $\left(\mathrm{DMRs}^{5}{ }_{\text {cis }}\right.$ and DMRs ${ }^{3.5}{ }_{\text {cis }}$ ) and trans $\left(\mathrm{DMRs}^{5}{ }_{\text {trans }}\right.$ and $\mathrm{DMRs} \mathrm{s}^{3.5}$ trans $)$ relative to the PGC loci, i.e., those that were within the GWAS LD blocks, and those outside these blocks (Fig. 4). First, by comparing the heritability estimates from cis versus trans gDMRs at both cutoffs (i.e., DMRs ${ }^{5}$ is versus DMRs ${ }^{5}$ trans), we found that the majority of schizophrenia heritability and enrichment was driven by cis regions. For example, among the DMRs ${ }^{3.5}$, the subset that was cis $\left(\mathrm{DMRs}^{3.5}{ }_{\text {cis }}\right.$ ) explained $12 \%$ of $h^{2} \mathrm{SNP}$, with a 156 -fold enrichment over the whole genome $(P=1.3 \mathrm{e}-14)$, and were further highly enriched compared to the background of the overall GWAS-significant LD blocks. Approximately $80 \%$ of all cis $h^{2}{ }_{\mathrm{SNP}}$ of the GWASsignificant loci (LD blocks) were captured by DMRs ${ }^{3.5}{ }_{\text {cis }}(12 \%$ versus $15 \%$ ), even though they contained only $3 \%$ of loci sequence $(1.65 \mathrm{Mb}$ versus $56.5 \mathrm{Mb})$. In contrast, DMRs ${ }^{3.5}$ trans only explained $1.7 \%$ of $h^{2} \mathrm{SNP}$, and were not significantly enriched for schizophrenia risk $((P=0.14)$; Fig. 4$)$. Despite representing a very small portion of the genome $(658 \mathrm{~kb})$, the more stringent cis gDMRs still explained $8.7 \%$ of $h^{2}{ }_{\mathrm{SNP}}$, with very strong enrichment (243-fold, $P=1 \mathrm{e}-10$ ). At only $48 \mathrm{~kb}$, the stringent trans gDMRs were not wide enough to effectively detect enrichment, and only explained $0.5 \%$ of $h^{2}$ SNP. These results together suggest that the majority of schizophrenia genetic risk in these genome-wide significant loci specifically localizes among small subsets of genomic regions associated with proximal/nearby DNAm levels.

Genetic regulation of $\mathrm{CpH}$ DNAm levels in homogenate brain tissue. While non-CpG $(\mathrm{CpH}) \mathrm{DNA}$ methylation predominantly occurs in neuronal cells in the human brain ${ }^{27}$, we could nevertheless observe detectable levels in homogenate/bulk tissue (which contains $20-40 \%$ neuronal cells ${ }^{44}$ ). We analyzed 64,806,159 CpH sites in the DLPFC and 34,909,109 CpH sites in the hippocampus, after filtering for only sites which had at least moderate coverage and non-zero methylation levels across samples (see "Methods" section). These numbers of observable sites in homogenate tissue from adult donors were much larger than in prenatal donors, as $\mathrm{CpH}$ methylation occurs in post-mitotic neurons ${ }^{45}$, and comparable to smaller studies of neuronal nuclei 


\begin{tabular}{ccccccc}
\hline Category & SNPs (\%) & $h^{2}(\%)$ & $\begin{array}{c}\text { Enrichment } \\
\text { (fold) }\end{array}$ & $\begin{array}{c}\text { P-value } \\
\text { (Enrichment) }\end{array}$ & Width (bp) & $\begin{array}{c}\text { P-value } \\
\text { (Coefficient) }\end{array}$ \\
\hline SCZD Loci & $1.491 \%$ & $15.0 \%$ & 10.1 & $8.01 \mathrm{e}-16$ & 56462978 & $3.32 \mathrm{e}-18$ \\
\hline $\mathrm{DMR}^{3.5}$ cis & $0.077 \%$ & $12.1 \%$ & 156.7 & $1.30 \mathrm{e}-14$ & 1658085 & $4.19 \mathrm{e}-17$ \\
$\mathrm{DMR}^{3.5}{ }_{\text {trans }}$ & $0.234 \%$ & $1.7 \%$ & 7.5 & 0.147 & 5427037 & 0.073142 \\
$\mathrm{DMR}_{\text {cis }}^{5}$ & $0.036 \%$ & $8.7 \%$ & 243.6 & $1.00 \mathrm{e}-10$ & 657860 & $4.36 \mathrm{e}-12$ \\
$\mathrm{DMR}_{\text {trans }}{ }^{2}$ & $0.003 \%$ & $0.5 \%$ & 181.2 & 0.036 & 48414 & 0.017321 \\
\hline
\end{tabular}

\section{GWAS}

Index SNP 1

Locus 1

-

Index SNP 2

Locus 2

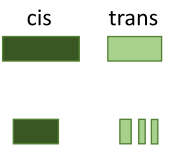

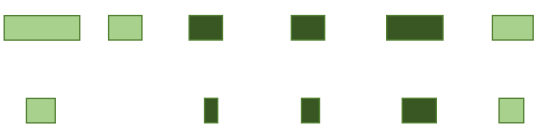

Fig. 4 LDSC results for schizophrenia heritability. (Top) LDSC analysis outputs for each category of gDMRs are explained here. Results showed that most of the enrichment for schizophrenia heritability in our gDMR sites was in cis. (Bottom) Visual description of LDSC: we performed LDSC analysis on the GWAS-identified loci as a background, and two sets of gDMRs, one with a cutoff of $t>3.5$ and one with a more stringent cutoff of $t>5$. We further divided these gDMR sets into cis gDMRs - those within the GWAS loci-and trans gDMRs-those outside the GWAS loci. $P$-values presented here are not adjusted for multiple testing, though multiple testing correction was used to determine significance in these analyses.

sorted with the NeuN antibody and subjected to $\mathrm{WGBS}^{46}$. We first performed full genome meQTL analysis on $\mathrm{CpH}$ sites, and found a robust presence of $\mathrm{CpH}-\mathrm{meQTL}$ in the DLPFC, with 25,584,299 SNP-CpH pairs representing 5,805,754 SNPs, 468,914 of which were not significant meQTLs for CpG sites (see "Data availability" section). These $\mathrm{CpH}$-associated SNPs further had CpG sites nearby, including in the testing window, suggesting potentially independent or complementary effects of $\mathrm{CpH}$ and CpG genetic associations. Unlike widespread $\mathrm{CpG}$ associations to genotype, there were far fewer unique $\mathrm{CpH}$ sites associated with genotype-only 976,094 $\mathrm{CpHs}$ associated with genotypes, corresponding to just $1.5 \%$ of tested sites. Generally, genetic control on $\mathrm{CpH}$ methylation appeared to have a narrower effect than on CpG methylation, with each SNP associating with a mean of 4 $\mathrm{CpH}$ sites over a mean width of $12,570 \mathrm{bp}$. The effect sizes of genotypes on methylation levels were much larger than they were for CpGs, with a mean of $27 \%$ change in methylation level per allele, and more than half $(57 \%)$ of these $\mathrm{CpH}$ sites were inside genes. The landscape of $\mathrm{CpH}$-meQTLs in the hippocampus was similar to DLPFC, identifying 25,043,471 SNP-CpH pairs, representing 5,853,364 SNPs and 781,490 $\mathrm{CpHs}$ (see "Data availability" section). A large majority (90\%)-but not all-of these SNPs and $63 \%$ of these CpH sites were also meQTLs in the DLPFC. Similarly, CpH-meQTLs had much larger effect sizes (mean $=29 \%$ ) and most represented $\mathrm{CpH}$ sites $(58 \%)$ were inside genes. These effects in each brain region presumably represent neuronal-specific genetic regulation of DNAm levels.

We also performed more focused $\mathrm{CpH}-\mathrm{meQTL}$ analyses on the PGC SNPs described above and found 1444 significant cis-meQTLs and 48 trans-meQTLs in the DLPFC (Supplementary Dataset 6). Again, a majority of PGC SNPs were represented (141/152). Some of these $\mathrm{CpH}$ sites were near CpG-meQTLs, but many were not (mean distance $=120 \mathrm{~kb}$, median $=2798 \mathrm{bp}$ ), suggesting potential independent effects of genotype on different sequence contexts of DNAm. Like with CpGs associated with genotype, $\mathrm{CpHs}$ in PGCmeQTLs were also enriched for transcriptional and weak transcriptional chromatin states over full genome $\mathrm{CpH}-\mathrm{meQTLs}$, and depleted for repressor polycomb and quiescent states
(Fig. 2c ${ }^{33}$. Most $\mathrm{CpHs}$ were inside genes that were subsequently enriched for neuronal GO terms related to neurons, synapses, and channels, further validating the neuronal contribution of $\mathrm{CpH}$ DNAm levels. We similarly observed much larger effect sizes of risk alleles in $\mathrm{CpHs}$ compared to CpGs in line with genome-wide analyses above, with a mean of $27 \%$ compared to $2 \%$, respectively. In the hippocampus, we found 1588 cis-CpH-meQTLs and 92 trans-CpH-meQTLs (Supplementary Dataset 7), representing 148/ 153 tested SNPs. Similar to all previous analyses we see that these sites are mostly inside genes and have much larger effect sizes than CpGs. The genes represented by these $\mathrm{CpHs}$ are enriched for GO terms related to neuronal anatomy, synapses, and IP3. Again, distance to the nearest CpG-meQTL is highly variable, ranging from 1 to 4,217,747 bp ( mean $=24,374$, median $=2,761)$. Results were overall similar between both brain regions, and $1219 \mathrm{CpH}-$ meQTLs were in common between both regions, though again, there were unique associations across regions. Overall, all PGC index SNPs were meQTLs, with most associating with both CpG and $\mathrm{CpH}$ sites, but a small percentage only associating with one cytosine context.

Age associations to DNAm levels. While there was extensive evidence of meQTLs in our WGBS data, there were a subset of CpG sites that showed high percentages of variance explained by age (Fig. 1f). We therefore more formally modeled methylation over age in both brain regions, as DNA methylation has been shown to globally accumulate with age $e^{77,48}$. We found an extensive association with age; nearly 1 million CpGs $(\mathrm{DLPFC}=765,861, \mathrm{HIPPO}=972,047)$ associated with age at FDR $<0.01$, and $\sim 2$ million CpG sites in each region (at FDR $<$ 0.05 , see "Data availability" section). The majority of these sites were age-associated in both regions, with a sizable fraction of sites showing some regional specificity $(700,000$ sites in the DLPFC and 800,000 sites in the hippocampus). The majority (94\%) of sites increase in methylation with age, with half of the sites in promoter regions, and a quarter in CpG islands or shores. Only $9 \%$ of genes represented by these differentially methylated 
promoters had a significant correlation to gene expression levels in these samples, perhaps resulting from DNAm and RNA-seq data being derived from different tissue dissections, and thus having the different cellular composition (see "Methods" section). In contrast, there was very little $\mathrm{CpH}$ association with age, with only 5136 and 445 significant sites in hippocampus and DLPFC respectively (at FDR $<0.05$ ). These results suggest that $\mathrm{CpH}$ methylation may be more stable across adulthood and aging after establishment in postnatal life.

Given the large extent of meQTL- and age-associated sites, we asked whether any $\mathrm{CpG}$ sites showed dynamic meQTL effects across the adult lifespan. Despite age being associated with methylation at many sites throughout the genome, we found there were practically no changes in meQTL effects across the adult lifespan (i.e., statistical interaction between age and genotype), and, if anything, sites that were differentially methylated by age were depleted $(P<2.2 \mathrm{e}-16)$ for being associated with local genetic variation (i.e., being meQTLs).

Minimal illness-state associated differential methylation levels. We lastly modeled methylation differences between patients with schizophrenia $\left(n_{\mathrm{DLPFC}}=70, n_{\mathrm{HIPPO}}=77\right)$ and neurotypical controls $\left(n_{\mathrm{DLPFC}}=95, n_{\mathrm{HIPPO}}=102\right)$. These associations are typically more subtle-fewer sites with smaller effect sizes-than age or genotype effects in microarray data ${ }^{10}$ and more likely to represent cohort- or data set-specific findings ${ }^{49}$. In these WGBS data, we found very few FDR-significant CpG sites-none in DLPFC and 70 in the hippocampus. This is perhaps not surprising based on previous studies and the high multiple testing burden-almost two orders of magnitude more than microarray platforms-and smaller sample sizes due to the expense and computational intensity of WGBS. Re-analysis of our previous DLPFC Illumina 450k microarray data limited to the 164 donors with WGBS data identified an order of magnitude fewer sites than in the full cohort (184 CpGs versus $2104 \mathrm{CpGs}$ at the same Bonferroni-corrected $P<0.05$ ), and further, only 4 sites in this reanalysis remained significant enforcing Bonferroni correction using the number of WGBS-tested (rather than microarray-tested) sites, emphasizing the importance of both of these factors in discovery. We found a similar lack of case-control signal at $\mathrm{CpH}$ sites, with no significant hits in DLPFC and 1293 in hippocampus, with most $(70 \%)$ of the hippocampal hits being in or nearby genes. These results suggest that despite major environmental associations of chronic schizophrenia, including smoking, drug treatment, general health deprivations, and chronic psychological stress, the effects observable on the methylome in bulk tissue are remarkably subtle, particularly in the contexts of much stronger genotype- and age-associated effects.

\section{Discussion}

Here we present the most comprehensive whole-genome bisulfite sequencing (WGBS) study-particularly in the human brain-to date, to better understand technical and biological factors that contribute to genome-wide DNA methylation levels at both CpG and $\mathrm{CpH}$ sites. We first demonstrated, at a single base-pair resolution, that meQTLs are highly abundant throughout the entire genome at a breadth and scope previously unseen. Not only can common SNPs associate with CpG methylation, but they also uniquely and independently associate with $\mathrm{CpH}$ methylation levels in adult neurons. Furthermore, we demonstrated the clinical relevance of these single base resolution meQTL maps to identify the functional significance of loci identified by GWAS in the human brain. Using schizophrenia as an example, we found DNA methylation associations to nearly every genome-wide significant variant that clustered into many local genetic differentially methylated regions (gDMRs) that explained significant proportions of disease heritability. We have further created a user-friendly meQTL browser so that other researchers may use this resource to better understand their own genomic regions of interest.

Due to the expense and computational intensity, WGBS is challenging for epigenomic studies. With our large-scale study, we were able to identify the effects of technical and potential biological variables on our data. This has been less well characterized than microarray studies, and we found that batch and ancestry cause much variance in the data, and their effects are exacerbated and alleviated, respectively, by the smoothing process. We also found that ENCODE blacklist regions are unreliable in WGBS data, due to the increased difficulty of alignment ${ }^{30}$. Overall, it is clear that genotype and age impact methylation at a large number of $\mathrm{CpG}$ and $\mathrm{CpH}$ sites, contrasting with schizophrenia disease state which associates very little with DNAm. There were few SCZD-associated CpGs in the hippocampus and none in DLPFC, potentially in contrast to previous work ${ }^{10}$. We note that our previous microarray study had a much larger sample size (191 cases, 240 controls), and identified CpG probes with very small differences in DNAm levels $(<0.05)$, which were likely smaller than the precision of WGBS with average post-QC coverage of 22 reads (even by increasing precision through smoothing).

Previous studies have identified a genomic presence of meQTLs, but not at a single base-pair resolution. Our findings are largely consistent with previous work, in that meQTLs are indeed extensive throughout the genome, and that most of their regulation occurs locally. However, while earlier estimates reported that $15 \%$ of $\mathrm{CpGs}$ were under genetic control ${ }^{11}$, we greatly increased this fraction to $55 \%$. Like Smith et al. ${ }^{8}$, we showed that overlap was generally high between the two brain regions we surveyed, though there are differences as well. Studies have also found that functional meQTLs are enriched for active chromatin states $^{11}$ and that meQTLs appear to impact alternative splicing ${ }^{12}$, further agreeing with our results and supporting the idea that schizophrenia risk associated loci may represent functional meQTLs. With our large sample and high genomic breadth, we are able to expand on all of these earlier findings at an in-depth genomic level.

These results further implicate DNAm as perhaps the most proximal molecular correlate of DNA sequence variation. The most comprehensive eQTL resource constructed in brain tissue, using over 1400 individuals, identified that $\sim 25 \%$ of common genetic variants associated with nearby gene expression levels ${ }^{50}$ and our meQTL maps here implicated three times as many SNPs (76\%) with a much smaller number of donors. Similarly, the recent GTEx v8 eQTL efforts-performed across 838 donors and 17,382 RNA-seq samples across 49 tissues-implicated $43 \%$ of tested SNVs with gene expression in at least one tissue.

These meQTLs further refined our understanding of the functional significance of schizophrenia genetic risk loci. By leveraging WGBS data combined with genotype data from the same samples, we identified molecular phenotypes associated with individual risk variants. This process could more generally filter GWAS findings to regions of the genome that could impart functional consequences of these risk variants. We found that regions that are differentially methylated by risk-associated genotypes explained most of the heritability imparted by the genomewide significant schizophrenia risk loci, despite spanning a much smaller fraction of the genome (1.6 versus $56.5 \mathrm{Mb})$. We also found that for some of these risk loci which have been previously identified as eQTLs ${ }^{39}$, DNA methylation mediates eQTL effects, refining the potential mechanism by which genetic risk variants may affect brain function. We note that the strongest mediation 
effects were seen among exons, indicating that differences in methylation may be key to alternative splicing, as has been previously hypothesized ${ }^{51}$. While our data do not show that DNAm mediates expression for the majority of the meQTLs, this must be viewed with some caution. Our brain samples represent a moment in time in the lifespan of any given brain, and the data are from bulk tissue. At different life stages, perhaps in specific cell populations, mediation effects may be more prominent, particularly in the developing brain ${ }^{28}$. The interplay between sequence variation, DNAm, and gene expression will likely be refined across cell type-specific analyses from the same source tissue.

WGBS also gives the unique ability to examine $\mathrm{CpH}$ methylation, an often overlooked mark, particularly in the brain. We found that DNAm levels at specific $\mathrm{CpH}$ sites were also associated with genetic variation, which presumably reflected neuronspecific genetic regulation of DNAm levels. It is interesting that the genetic control of $\mathrm{CpH}$ methylation seems to have a much larger effect size than that on $\mathrm{CpGm}$, particularly given the fact that the fraction of neurons in our homogenate tissue were uniquely driving these associations. This mark is particularly interesting to examine in relation to psychiatric disorders because it is specific to neurons, so we can point to the cell type of interest at these sites. Understanding which $\mathrm{CpHs}$ are under the control of risk loci even further refines our understanding of the risk loci's functions because of this. It is also interesting that despite $\mathrm{CpHs}$ being abundant throughout the genome, most meQTL$\mathrm{CpHs}$ are inside genes, possibly further pointing to functional significance. Large-scale analyses in sorted neuronal cell populations can further refine these associations, particularly in different subpopulations of neurons (i.e., inhibitory and excitatory) ${ }^{52}$.

Overall, we have established a comprehensive landscape of genetic control of genomic methylation in the human brain. Based on previous findings that many meQTLs are stable across tissue types, a large fraction of this meQTL map could apply to other tissues and cell types. It is clear that genotype has a robust role in determining local methylation levels, not only at $\mathrm{CpG}$ sites but at $\mathrm{CpH}$ sites as well. These findings can further be applied to understand the functional significance of genetic risk loci identified in GWAS.

\section{Methods \\ Study samples. Postmortem brain specimens were donated through the Offices of the Chief Medical Examiners of the District of Columbia and of the Common- wealth of Virginia, Northern District to the NIMH Brain Tissue Collection at the National Institutes of Health in Bethesda, MD, according to NIH Institutional Review Board guidelines (Protocol \#90-M-0142). Audiotaped informed consent was obtained from legal next-of-kin on every case (as these donations occurred after death, and thus the donors themselves could not consent). Details of the donation process are described previously ${ }^{53,54}$. All adult neurotypical controls were free from psychiatric and/or neurologic diagnoses and substance abuse according to DSM-IV, and had toxicology screening to exclude acute drug and alcohol intoxication/use at the time of death ${ }^{55}$.}

WGBS data generation. Genomic DNA was extracted from $100 \mathrm{mg}$ of the pulverized dorsolateral prefrontal cortex (DLPFC, corresponding to BA46/9) or hippocampus tissue (dissected as previously described ${ }^{39}$ ) with the phenol-chloroform method. The hippocampus was dissected from the anterior tip posteriorly through to the midbody of the hippocampus at the level of the lateral geniculate nucleus, which included the hippocampus proper (i.e., Ammons Horn and CA1-3) plus the subicular complex. The DLPFC was dissected in a plane perpendicular to the pial surface in area 46 of the cortex to capture from the pial surface to the gray matter-WM junction.

DNA was subjected to bisulfite conversion followed by sequencing library preparation using the TruSeq DNA methylation kit from Illumina. Lambda DNA was spiked prior to bisulfite conversion to assess its rate, and we used $20 \%$ PhiX to better calibrate Illumina base calling on these lower complexity libraries. The resulting libraries were pooled and sequenced on an Illumina HiSeq X Ten sequencer with paired-end $150 \mathrm{bp}$ reads $(2 \times 150 \mathrm{bp})$, targeting $90 \mathrm{~Gb}$ per sample. This corresponds to $30 \times$ coverage of the human genome as extra reads were generated to account for the addition of PhiX.
Data processing. The raw WGBS data was processed using FastQC to control for quality of reads, Trim Galore to trim reads and remove adapter content ${ }^{56}$, Arioc for alignment to the GRCh38.p12 genome (obtained from https://ftp.ncbi.nlm.nih.gov/ genomes/all/GCA/000/001/405/GCA_000001405.27_GRCh38.p12/ GCA 000001405.27_GRCh38.p12 assembly_structure/Primary_Assembly/ assembled_chromosomes $/)^{57}$, duplicate alignments were removed with SAMBLASTER $^{58}$, and filtered with samtools ${ }^{59}$ (v1.9) to exclude all but primary alignments with a MAPQ $\geq 5$. We used the Bismark methylation extractor to extract methylation data from aligned, filtered reads ${ }^{60}$. We then used the bsseq $\mathrm{R} /$ Bioconductor package (v1.18) to process and combine the DNA methylation proportions across the samples for all further manipulation and analysis ${ }^{31}$. After initial data metrics were calculated, the methylation data for each sample was locally smoothed using BSmooth with default parameters for downstream analyses. CpG results were filtered to those, not in blacklist regions (DLPFC $N=26,155,085$, Hippocampus $N=26,301,249$ ), and those which had coverage $\geq 3$. CpHs were filtered to sites that had $>3$ coverage and non-zero methylation in at least half the samples. Due to an unidentifiable primary source of variance, 11 samples in the DLPFC were dropped before analysis. We also extracted DNA sequence variants from 740 common exonic/coding sites for comparisons to DNA genotyping data to confirm sample identities, as implemented in our SPEAQeasy RNA-seq software ${ }^{61}$.

DNA SNP genotyping. Genotype data on the 183 unique donors under study were processed and imputed with additional donors across the full LIBD postmortem genotype data ${ }^{18}$. Genetic imputation was performed on high-quality observed genotypes (removing low quality and rare variants) using the prephasing/imputation stepwise approach implemented in IMPUTE2 ${ }^{62}$ and Shape-IT ${ }^{63}$ imputation reference set from the full 1000 Human Genomes Project Phase 3 dataset $^{64}$ genome build hg19. Imputation was performed separately by the Illumina microarray platform across the entire brain collection, and study-specific samples were extracted from the imputed genotypes. This study contained three imputation batches, corresponding to the Illumina h650 $(N=43), 1 \mathrm{M}$ Duo $(N=139)$, and Omni5 $(N=2)$ platforms. Imputed genotypes for the entire cohort were merged across imputation runs/batches in the Oxford file format as dosages, then converted to plink file format as "hard call" genotypes (treating variants with posterior probabilities $<0.9$ as missing). After filtering to just the donors in this study, we retained common variants (MAF $>5 \%$, relative to this sample) that were present in the majority of samples (missingness $<10 \%$ ) and that were in Hardy-Weinberg equilibrium (at $P>1 \times 10^{-6}$ ) using the Plink tool kit version $1.90 \mathrm{~b} 3 \mathrm{a}^{65}$. Multidimensional scaling (MDS) was performed on autosomal LD-independent SNPs (variation inflation factor $=1.25$, corresponding to $R^{2}<0.2$ ) to construct genomic ancestry components on each sample, which can be interpreted as quantitative levels of ethnicity-the first component separated the European and African American samples, for inclusion as potential confounders in the differential methylation analyses described below. We also extracted 740 observed and imputed DNA-genotyped SNPs, and successfully confirmed sample identities against these same variants extracted from the WGBS data.

Assessment of technical and biological variation. Principal component analyses (PCA) were performed on the $1 \mathrm{e} 6$ most variable autosomal $\mathrm{CpG}$ sites using the $\operatorname{prcomp}()$ function in $\mathrm{R}$. We calculated the percentage of variance explained by biological and technical variables using the anova() and $\operatorname{lm}()$ functions in $\mathrm{R}$.

meQTL analysis. We used R package Matrix eQTL ${ }^{66}$ (v2.3) in all meQTL analyses For full genome analysis, we set the maximum cis SNP to "gene" distance to $20 \mathrm{~kb}$. We approximated the $P$-value equivalent to FDR $=0.01$ and used this as the $P$ value cutoff. We used only SNPs which were common (with minor allele frequencies, $\mathrm{MAF}>5 \%$ ) across the donors in each data set separately that were in Hardy-Weinberg equilibrium (at $P>1 \mathrm{e}-6)$ with high non-missingness $(>90 \%$ present), leading to analysis of 7,897,043 SNPs in the DLPFC and 7,865,986 SNPs in the hippocampus. The model adjusted for 28 covariates, which were the top 28 principal components of the methylation data. For PGC SNP analyses, we set the cis distance to $250 \mathrm{~kb}$, and considered everything else in trans. We set the $P$-value cutoff to 1 so that we had statistics for every SNP-CpG pair in this analysis. meQTL interaction with age, neuronal composition, and MDS1 was assessed using the modelLINEAR_CROSS parameter. meQTLs were then organized into gDMRs by using the bumphunter R/Bioconductor package (v1.30) ${ }^{67}$ function regionFinder to create clusters of adjacent meQTLs which all had an association statistic of $t \geq 5$. These were filtered to gDMRs containing at least two adjacent CpGs. We used the cleaningY() function from the jaffelab package ${ }^{68}$ version 0.99 .20 to regress out adjustment covariates to visualize the DNAm levels in subsequent plots. In order to assess replicability and consistency between meQTL analyses and different sample subgroups, we calculated $\pi_{1}$ statistics, which estimates the fraction of alternative hypotheses. To do this, we take all significant meQTLs from the main analysis, and then calculate the statistics for these SNP-CpG pairs in the data set we wish to compare. We then take all the $P$-values-regardless of their significance-from the comparison group, and use R package $q$-value ${ }^{69}$ (v2.20) to calculate $\pi_{0}$ (which is the proportion of true null hypotheses), which is then subtracted from 1 to calculate $\pi_{1}$. 
Heritability analysis. We estimated the SNP-heritability of DNAm for each CpG site using the GCTA software ${ }^{35}$. We removed seven samples of DLPFC and eight samples of HIPPO so that all pairs of retained samples (DLPFC 158, HIPPO 171) had a genetic relatedness less than 0.025 and were included for heritability estimation. The genetic relationship matrix (GRM) was calculated using SNPs around each CpG site at three different window sizes $(40 \mathrm{~kb}, 200 \mathrm{~kb}$, and $1 \mathrm{Mb})$. We included the same set of covariates as we used in meQTL analysis in heritability estimation.

Functional significance analysis. We annotated our data to nearby genes relative to Gencode v. 29 on hg38. We performed Gene Ontology and gene set enrichment using clusterProfiler $(\mathrm{v} 3.12)^{70}$ with a $P$-value cutoff of 0.01 and $q$-value cutoff of 0.05 .

Stratified linkage disequilibrium score regression. We performed stratified LDscore regression (LDSC) as described by Finucane et al ${ }^{43}$, as implemented by Rizzardi, Hickey et al. ${ }^{71}$ for defined DMRs using summary statistics from recent GWAS $^{72}$. More detailed methods are provided in Price, Collado-Torres, et al. ${ }^{46}$, including code: https://github.com/LieberInstitute/brain-epigenomics/tree/master/ LDSC/code. Briefly, we used LDSC (LD SCore) v1.0.0 to estimate the proportion of heritability captured in sets of gDMRs for each GWAS phenotype, as well as central nervous system annotations included in the LDSC package (referred to as CNS (LDSC)), and regions annotated as putatively regulatory in the human brain using chromHMM (i.e., the union of regions annotated as "Bivalent Enhancer," "Bivalent/Poised TSS," "Genic enhancers," "Flanking Active TSS," "Active TSS," "Strong transcription," and "Enhancers"). After converting the GWAS summary statistics into the .sumstats format using munge_sumstats.py, we filtered to only HapMap 3 SNPs (downloaded from https://data.broadinstitute.org/alkesgroup/LDSCORE/ w_hm3.snplist.bz2) as described in the Partitioned Heritability LDSC tutorial. The partitioned heritability for each gDMR-GWAS combination by adding each feature individually to the "baseline model" including 53 baseline annotations described in Finucane et al. ${ }^{43}$.

DNA methylation mediation of expression. To assess mediation of gene expression, we identified SNPs which were both eQTLs ${ }^{39}$ and meQTLs, and which had some correlation $($ cor $>0.3)$ between gene expression and methylation levels. For every CpG-gene pair generated by this, we modeled the effect of additive genotype on expression (Expression $\sim$ SNP), then added in the effect of methylation (Expression $\sim$ SNP + DNAm), and examined the difference in the effect size/ coefficient for genotype between the two models. To determine the extent of mediation, we calculated the ratio of SNP coefficient in the second model to the SNP coefficient in the first. When the proportion was $<75 \%$ ( $>25 \%$ reduction), we considered this evidence of mediation. The same analysis was done for exon and junction expression data and their significant eQTLs.

Age and diagnosis differential methylation modeling. Differential methylation analyses for both diagnosis and age were performed using linear regression modeling, accounting for sex, estimated neuronal fraction, batch, and the top three MDS components from genotype data. The regression analyses above were formed using limma (v3.30 $)^{73,74}$ which employed empirical Bayes and returned moderated T-statistics, which were used to calculate $P$-values and estimate the false discovery rate (FDR, via Benjamini-Hochberg approach ${ }^{75}$ ).

General statistical reporting. Sample sizes were 165 samples in DLPFC analysis, 179 samples in hippocampus analysis, and 344 samples in combined analyses (161 DLPFC and hippocampus matched pairs from the same donors). All box plots shown in the main and supplementary figures display the median as the center, IQR (25th-75th percentile) as the box range, and 1.5 times the IQR as the whiskers. All reported $P$-values are two-sided, and multiple testing correction method is noted in the text. Distributions of the residuals of our many linear models were assumed to be normally distributed across all sites and models, but this was not formally tested. All points were used in all analyses: for example, outliers were not removed.

Reporting summary. Further information on research design is available in the Nature Research Reporting Summary linked to this article.

\section{Data availability}

We have created a user-friendly and fast meQTL browser that allows searching by SNPs or cytosines by genomic regions (chr.start-end) at https://eqtl.brainseq.org/WGBS_meQTL/. Raw and processed nucleic acid sequencing data generated to support the findings of this study are available via the PsychENCODE Knowledge Portal (https:// psychencode.synapse.org/). The PsychENCODE Knowledge Portal is a platform for accessing data, analyses, and tools generated through grants funded by the National Institute of Mental Health (NIMH) PsychENCODE program. Data is available for general research use according to the following requirements for data access and data attribution: (https://psychencode.synapse.org/DataAccess). For access to content described in this manuscript see: https://doi.org/10.7303/syn25992404. Full results data sets can be found in the same repository or in the Supplement's "Description of Additional Supplementary Files". Due to containing identifiable information, genotype data are available through controlled access via the corresponding authors following successful access to $\mathrm{dbGaP}$ data set phs000979.

\section{Code availability}

Analysis code that accompanies this paper is provided on GitHub (https://github.com/ LieberInstitute/wgbs_meqtl_analysis) and available at Zenodo (https://doi.org/10.5281/ zenodo. 5113698$)^{76}$.

Received: 24 March 2021; Accepted: 12 August 2021; Published online: 02 September 2021

\section{References}

1. Heijmans, B. T. et al. Persistent epigenetic differences associated with prenatal exposure to famine in humans. Proc. Natl Acad. Sci. USA 105, 17046-17049 (2008).

2. Breitling, L. P., Yang, R., Korn, B., Burwinkel, B. \& Brenner, H. Tobaccosmoking-related differential DNA methylation: $27 \mathrm{~K}$ discovery and replication. Am. J. Hum. Genet. 88, 450-457 (2011).

3. Davies, M. N. et al. Functional annotation of the human brain methylome identifies tissue-specific epigenetic variation across brain and blood. Genome Biol. 13, R43 (2012)

4. Kaminsky, Z. A. et al. DNA methylation profiles in monozygotic and dizygotic twins. Nat. Genet. 41, 240-245 (2009)

5. Bell, J. T. \& Spector, T. D. DNA methylation studies using twins: what are they telling us? Genome Biol. 13, 172 (2012)

6. Meaburn, E. L., Schalkwyk, L. C. \& Mill, J. Allele-specific methylation in the human genome: implications for genetic studies of complex disease. Epigenetics 5, 578-582 (2010).

7. Sved, J. \& Bird, A. The expected equilibrium of the CpG dinucleotide in vertebrate genomes under a mutation model. Proc. Natl Acad. Sci. USA 87, 4692-4696 (1990).

8. Smith, A. K. et al. Methylation quantitative trait loci (meQTLs) are consistently detected across ancestry, developmental stage, and tissue type. BMC Genomics 15, 145 (2014).

9. Schulz, H. et al. Genome-wide mapping of genetic determinants influencing DNA methylation and gene expression in human hippocampus. Nat. Commun. 8, 1511 (2017).

10. Jaffe, A. E. et al. Mapping DNA methylation across development, genotype and schizophrenia in the human frontal cortex. Nat. Neurosci. 19, 40-47 (2016).

11. McClay, J. L. et al. High density methylation QTL analysis in human blood via next-generation sequencing of the methylated genomic DNA fraction. Genome Biol. 16, 291 (2015).

12. Gutierrez-Arcelus, M. et al. Tissue-specific effects of genetic and epigenetic variation on gene regulation and splicing. PLoS Genet. 11 e1004958 (2015).

13. Hoffmann, A., Ziller, M. \& Spengler, D. The future is the past: methylation qtls in schizophrenia. Genes 7, 104 (2016).

14. Gejman, P. V., Sanders, A. R. \& Duan, J. The role of genetics in the etiology of schizophrenia. Psychiatr. Clin. North Am. 33, 35-66 (2010).

15. Pardiñas, A. F. et al. Common schizophrenia alleles are enriched in mutationintolerant genes and in regions under strong background selection. Nat. Genet. 50, 381-389 (2018).

16. Schizophrenia Working Group of the Psychiatric Genomics Consortium, Ripke, S., Walters, J. T. \& O'Donovan, M. C. Mapping genomic loci prioritises genes and implicates synaptic biology in schizophrenia. Preprint at medRxiv https://doi.org/10.1101/2020.09.12.20192922 (2020).

17. Schrode, N. et al. Synergistic effects of common schizophrenia risk variants. Nat. Genet. 51, 1475-1485 (2019).

18. Jaffe, A. E. et al. Developmental and genetic regulation of the human cortex transcriptome illuminate schizophrenia pathogenesis. Nat. Neurosci. 21, $1117-1125$ (2018)

19. Fromer, M. et al. Gene expression elucidates functional impact of polygenic risk for schizophrenia. Nat. Neurosci. 19, 1442-1453 (2016).

20. Zhu, Z. et al. Integration of summary data from GWAS and eQTL studies predicts complex trait gene targets. Nat. Genet. 48, 481-487 (2016).

21. Gusev, A. et al. Transcriptome-wide association study of schizophrenia and chromatin activity yields mechanistic disease insights. Nat. Genet. 50, 538-548 (2018).

22. Gamazon, E. R. et al. A gene-based association method for mapping traits using reference transcriptome data. Nat. Genet. 47, 1091-1098 (2015). 
23. Huan, T. et al. Genome-wide identification of DNA methylation QTLs in whole blood highlights pathways for cardiovascular disease. Nat. Commun. 10, 4267 (2019).

24. Gamazon, E. R. et al. Enrichment of cis-regulatory gene expression SNPs and methylation quantitative trait loci among bipolar disorder susceptibility variants. Mol. Psychiatry 18, 340-346 (2013).

25. Baccarelli, A. \& Bollati, V. Epigenetics and environmental chemicals. Curr. Opin. Pediatr. 21, 243-251 (2009).

26. Dempfle, A. et al. Gene-environment interactions for complex traits: definitions, methodological requirements and challenges. Eur. J. Hum. Genet. 16, 1164-1172 (2008).

27. Guo, J. U. et al. Distribution, recognition and regulation of non-CpG methylation in the adult mammalian brain. Nat. Neurosci. 17, 215-222 (2014).

28. Birnbaum, R. \& Weinberger, D. R. Genetic insights into the neurodevelopmental origins of schizophrenia. Nat. Rev. Neurosci. 18, 727-740 (2017).

29. Fortin, J.-P., Triche, T. J. \& Hansen, K. D. Preprocessing, normalization and integration of the Illumina HumanMethylationEPIC array with minfi. Bioinformatics 33, 558-560 (2017).

30. Amemiya, H. M., Kundaje, A. \& Boyle, A. P. The ENCODE blacklist: identification of problematic regions of the genome. Sci. Rep. 9, 9354 (2019).

31. Hansen, K. D., Langmead, B. \& Irizarry, R. A. BSmooth: from whole genome bisulfite sequencing reads to differentially methylated regions. Genome Biol. 13, R83 (2012)

32. GTEx Consortium. The GTEx Consortium atlas of genetic regulatory effects across human tissues. Science 369, 1318-1330 (2020).

33. Roadmap Epigenomics Consortium et al. Integrative analysis of 111 reference human epigenomes. Nature 518, 317-330 (2015)

34. Kosoy, R. et al. Ancestry informative marker sets for determining continental origin and admixture proportions in common populations in America. Hum. Mutat. 30, 69-78 (2009).

35. Yang, J., Lee, S. H., Goddard, M. E. \& Visscher, P. M. GCTA: a tool for genome-wide complex trait analysis. Am. J. Hum. Genet. 88, 76-82 (2011).

36. Liu, Y. et al. Epigenome-wide association data implicate DNA methylation as an intermediary of genetic risk in rheumatoid arthritis. Nat. Biotechnol. 31, 142-147 (2013).

37. Feinberg, A. P. The key role of epigenetics in human disease prevention and mitigation. N. Engl. J. Med. 378, 1323-1334 (2018).

38. Ventham, N. T. et al. Integrative epigenome-wide analysis demonstrates that DNA methylation may mediate genetic risk in inflammatory bowel disease. Nat. Commun. 7, 13507 (2016).

39. Collado-Torres, L. et al. Regional heterogeneity in gene expression, regulation, and coherence in the frontal cortex and hippocampus across development and schizophrenia. Neuron 103, 203-216.e8 (2019).

40. Shukla, S. et al. CTCF-promoted RNA polymerase II pausing links DNA methylation to splicing. Nature 479, 74-79 (2011).

41. Bird, A. Perceptions of epigenetics. Nature 447, 396-398 (2007).

42. Jones, P. A. \& Taylor, S. M. Cellular differentiation, cytidine analogs and DNA methylation. Cell 20, 85-93 (1980).

43. Finucane, H. K. et al. Partitioning heritability by functional annotation using genome-wide association summary statistics. Nat. Genet. 47, 1228-1235 (2015).

44. Guintivano, J., Aryee, M. J. \& Kaminsky, Z. A. A cell epigenotype specific model for the correction of brain cellular heterogeneity bias and its application to age, brain region and major depression. Epigenetics 8, 290-302 (2013).

45. Perzel Mandell, K. A. et al. Characterizing the dynamic and functional DNA methylation landscape in the developing human cortex. Epigenetics 1-13, https://doi.org/10.1080/15592294.2020.1786304 (2020).

46. Price, A. J. et al. Divergent neuronal DNA methylation patterns across human cortical development reveal critical periods and a unique role of $\mathrm{CpH}$ methylation. Genome Biol. 20, 196 (2019).

47. Issa, J.-P. Age-related epigenetic changes and the immune system. Clin. Immunol. 109, 103-108 (2003)

48. Fraga, M. F., Agrelo, R. \& Esteller, M. Cross-talk between aging and cancer: the epigenetic language. Ann. N. Y. Acad. Sci. 1100, 60-74 (2007).

49. Jaffe, A. E. \& Kleinman, J. E. Genetic and epigenetic analysis of schizophrenia in blood-a no-brainer? Genome Med. 8, 96 (2016).

50. Wang, D. et al. Comprehensive functional genomic resource and integrative model for the human brain. Science 362, eaat8464 (2018).

51. Lev Maor, G., Yearim, A. \& Ast, G. The alternative role of DNA methylation in splicing regulation. Trends Genet. 31, 274-280 (2015).

52. Kozlenkov, A. et al. A unique role for DNA (hydroxy)methylation in epigenetic regulation of human inhibitory neurons. Sci. Adv. 4, eaau6190 (2018).

53. Lipska, B. K. et al. Critical factors in gene expression in postmortem human brain: Focus on studies in schizophrenia. Biol. Psychiatry 60, 650-658 (2006).

54. Deep-Soboslay, A. et al. Reliability of psychiatric diagnosis in postmortem research. Biol. Psychiatry 57, 96-101 (2005).
55. BrainSeq Consortium. Brainseq: neurogenomics to drive novel target discovery for neuropsychiatric disorders. Neuron 88, 1078-1083 (2015).

56. Krueger, F. TrimGalore: a wrapper around Cutadapt and FastQC to consistently apply adapter and quality trimming to FastQ files, with extra functionality for RRBS data. https://github.com/FelixKrueger/TrimGalore (2020).

57. Wilton, R., Li, X., Feinberg, A. P. \& Szalay, A. S. Arioc: GPU-accelerated alignment of short bisulfite-treated reads. Bioinformatics 34, 2673-2675 (2018).

58. Faust, G. G. \& Hall, I. M. SAMBLASTER: fast duplicate marking and structural variant read extraction. Bioinformatics 30, 2503-2505 (2014).

59. $\mathrm{Li}, \mathrm{H}$. et al. The sequence Alignment/Map format and SAMtools. Bioinformatics 25, 2078-2079 (2009).

60. Krueger, F. \& Andrews, S. R. Bismark: a flexible aligner and methylation caller for Bisulfite-Seq applications. Bioinformatics 27, 1571-1572 (2011).

61. Eagles, N. J. et al. SPEAQeasy: a scalable pipeline for expression analysis and quantification for R/bioconductor-powered RNA-seq analyses. BMC Bioinformatics 22, 224 (2021).

62. Howie, B. N., Donnelly, P. \& Marchini, J. A flexible and accurate genotype imputation method for the next generation of genome-wide association studies. PLoS Genet. 5, e1000529 (2009).

63. Delaneau, O., Coulonges, C. \& Zagury, J.-F. Shape-IT: new rapid and accurate algorithm for haplotype inference. BMC Bioinformatics 9, 540 (2008).

64. 1000 Genomes Project Consortium et al. A global reference for human genetic variation. Nature 526, 68-74 (2015).

65. Purcell, S. et al. PLINK: a tool set for whole-genome association and population-based linkage analyses. Am. J. Hum. Genet. 81, 559-575 (2007).

66. Shabalin, A. A. Matrix eQTL: ultra fast eQTL analysis via large matrix operations. Bioinformatics 28, 1353-1358 (2012).

67. Jaffe, A. E. et al. Bump hunting to identify differentially methylated regions in epigenetic epidemiology studies. Int. J. Epidemiol. 41, 200-209 (2012).

68. Collado-Torres, L. \& Jaffe, A. E. jaffelab: Commonly Used Functions by the Jaffe Lab (GitHub, 2018).

69. Storey, J. D. \& Tibshirani, R. Statistical significance for genomewide studies. Proc. Natl Acad. Sci. USA 100, 9440-9445 (2003).

70. Yu, G., Wang, L.-G., Han, Y. \& He, Q.-Y. clusterProfiler: an R package for comparing biological themes among gene clusters. OMICS 16, 284-287 (2012).

71. Rizzardi, L. F. et al. Neuronal brain-region-specific DNA methylation and chromatin accessibility are associated with neuropsychiatric trait heritability. Nat. Neurosci. 22, 307-316 (2019).

72. Brainstorm Consortium et al. Analysis of shared heritability in common disorders of the brain. Science 360, eaap8757 (2018).

73. Ritchie, M. E. et al. limma powers differential expression analyses for RNAsequencing and microarray studies. Nucleic Acids Res. 43, e47 (2015).

74. Phipson, B., Lee, S., Majewski, I. J., Alexander, W. S. \& Smyth, G. K. Robust hyperparameter estimation protects against hypervariable genes and improves power to detect differential expression. Ann. Appl. Stat. 10, 946-963 (2016).

75. Benjamini, Y. \& Hochberg, Y. Controlling the false discovery rate: A practical and powerful approach to multiple testing. J. R. Stat. Soc. 57, 289-300 (1995).

76. Kperzel. LieberInstitute/wgbs_meqtl_analysis: publication version. Zenodo https://doi.org/10.5281/zenodo.5113698 (2021)

\section{Acknowledgements}

We would like to express their gratitude to our colleagues whose tireless efforts have led to the donation of postmortem tissue to advance these studies: the Office of the Chief Medical Examiner of the District of Columbia; the Office of the Chief Medical Examiner for Northern Virginia, Fairfax Virginia; and the Office of the Chief Medical Examiner of the State of Maryland, Baltimore, Maryland. We would also like to acknowledge Llewellyn B. Bigelow, MD, for his diagnostic expertise. This project was supported by The Lieber Institute for Brain Development and by NIH grants R01MH112751 and T32GM781437. Finally, we are indebted to the generosity of the families of the decedents, who donated the brain tissue used in these studies.

\section{Author contributions}

K.A.P.M. and A.E.J. conceptualized the project and methodology, investigated and analyzed the data, and wrote the paper. S.H. aided in analysis. W.S.U., L.C.-T., A.S.S., R.W. and N.J.E. provided software support. W.S.U. created the online meQTL browser N.J.E., A.J.P., R.T., T.M.H. and R.W. curated data. J.E.K., R.T. and T.M.H. provided resources. S.H., A.J.P., L.C.-T., S.A.S., A.E.J. and D.R.W. reviewed and edited the paper D.R.W. provided supervision, and A.E.J. was the principal investigator and oversaw experimental design and analysis. All authors read and approved the final manuscript.

\section{Competing interests}

Andrew E. Jaffe is now employed by a for-profit biotechnology start-up company (with company name pending), which is unrelated to the contents of this manuscript. The remaining authors declare no competing interests. 


\section{Additional information}

Supplementary information The online version contains supplementary material available at https://doi.org/10.1038/s41467-021-25517-3.

Correspondence and requests for materials should be addressed to D.R.W. or A.E.J.

Peer review information Nature Communications thanks Irina Voineagu and the other anonymous reviewer(s) for their contribution to the peer review of this work. Peer reviewer reports are available.

Reprints and permission information is available at http://www.nature.com/reprints

Publisher's note Springer Nature remains neutral with regard to jurisdictional claims in published maps and institutional affiliations. (c) (P) Open Access This article is licensed under a Creative Commons Attribution 4.0 International License, which permits use, sharing, adaptation, distribution and reproduction in any medium or format, as long as you give appropriate credit to the original author(s) and the source, provide a link to the Creative Commons license, and indicate if changes were made. The images or other third party material in this article are included in the article's Creative Commons license, unless indicated otherwise in a credit line to the material. If material is not included in the article's Creative Commons license and your intended use is not permitted by statutory regulation or exceeds the permitted use, you will need to obtain permission directly from the copyright holder. To view a copy of this license, visit http://creativecommons.org/ licenses/by/4.0/.

(C) The Author(s) 2021 